\title{
Hyperstability and Stability of a Logarithm-type Functional Equation
}

\author{
Young Whan Lee ${ }^{1}$, Gwang Hui Kim ${ }^{2, *}$ \\ ${ }^{1}$ Department of Computer Hacking and Information Security, College of Engineering, Daejeon University, Daejeon, 34520, Korea \\ ${ }^{2}$ Department of Mathematics, Kangnam University, Yongin, Gyeonggi, 16979, Korea
}

Copyright (C) 2019 by authors, all rights reserved. Authors agree that this article remains permanently open access under the terms of the Creative Commons Attribution License 4.0 International License

\begin{abstract}
In 2001, Maksa and Páles [12] introduced a new type's stability: hyperstability for a class of linear functional equation $f(x)+f(y)=\frac{1}{n} \sum_{i=1}^{n} f\left(x \varphi_{i}(y)\right)$. Riedel and Sahoo [14] have generalized a functional equation associated with the distance between the probability distributions, which is $f(p r, q s)+f(p s, q r)=2 M(r s) f(p, q)+2 M(p q) f(r, s)$. Elfen etc. [7] obtained the solution of the functional equation $f(p r, q s)+f(p s, q r)=2 f(p, q)+2 f(r, s)$ on semigroup $G$. The aim of this paper is to investigate the hyperstability and the Hyers-Ulam stability for the above Logarithm-type functional equation considered by Elfen, etc. Namely, if $f$ is an approximative equation related to the above equation, then it is a solution of this equation which exists within $\varepsilon-$ bound of a given approximative function $f$.
\end{abstract}

Keywords Information Measure, Distance Measure, Superstability, Multiplicative Function, Stability of Functional Equation

AMS Subject Classification: 39B82, 39B52

\section{Introduction}

The following stability problem is well-known as Ulam's stability problem [16]:

Let $G_{1}$ be a group and let $G_{2}$ be a metric group with a metric $d(\cdot, \cdot)$. Given $\epsilon>0$, does there exist a $\delta>0$ such that if a mapping $h: G_{1} \rightarrow G_{2}$ satisfies the inequality $d(h(x y), h(x) h(y))<\delta$ for all $x, y \in G_{1}$, then there exists a homomorphism $H: G_{1} \rightarrow G_{2}$ with $d(h(x), H(x))<\epsilon$ for all $x \in G_{1}$ ?

In next year, Hyers [11] proved a first partial answer to Ulam's problem for an additive mapping on a Banach space. D. G. Bourgin obtained many excellent results for the stability ([3], [4]).Hyers' theorem was generalized by Aoki [1] for the case bounded by variables, and their results are improved by Rassias [13] to the case of the linear mapping and by Ger [9] . Găvruta [8] proved a further generalization of the Rassias' theorem by using a general control function.

The superstability phenomenon of the exponential equation $f(x+y)=f(x) f(y)$ was discovered by Baker, Lawrence, and Zorzitto [2] in 1979. The superstability for asymptotic phenomenon of the exponential equation was discovered by Ger [9].

In 2001, Maksa and Páles [12] proved a new type's stability for a class of linear functional equation

$$
f(x)+f(y)=\frac{1}{n} \sum_{i=1}^{n} f\left(x \varphi_{i}(y)\right),
$$

where $f$ is a real-valued mapping defined on a semigroup $S$, and the mappings $\varphi_{1}, \varphi_{2}, \cdots, \varphi_{n}: S \rightarrow S$ are pairwise distinct automorphisms. That is as following:

Let $\varepsilon: S \times S \rightarrow \mathbb{R}$ be a function such that there exists a sequence $u_{k}$ that satisfies

$$
\lim _{k \rightarrow \infty} \varepsilon\left(u_{k} s, t\right)=0 \quad(s, t \in S) .
$$

Assume that $f: S \rightarrow X$ satisfies the stability inequality

$$
\left\|f(s)+f(t)-\frac{1}{n} \sum_{i=1}^{n} f\left(s \varphi_{i}(t)\right)\right\| \leq \varepsilon(s, t) \quad(s, t \in S),
$$


where $X$ is a real normed space. Then, $f$ is a solution of (1).

Such a phenomenon is called the hyperstability of the functional equation. Gselmann [10], Brazdęk and Ciepliński [5] investigated the hyperstability of functional equations. A similar concept was introduced by Sirouni and Kabbaj [15].

Riedel and Sahoo [14] solved a functional equation associated with the distance between the probability distributions. Let $M:(0.1) \rightarrow \mathbb{C}$ be a given multiplicative function. Then, if $f:(0.1)^{2} \rightarrow \mathbb{C}$ satisfies the functional equation

$$
f(p r, q s)+f(p s, q r)=2 M(r s) f(p, q)+2 M(p q) f(r, s)
$$

if and only if

$$
f(p, q)=M(p) M(q)\left[L(p)+L(q)+l\left(\frac{p}{q}, \frac{p}{q}\right)\right]
$$

where $M:(0.1) \rightarrow \mathbb{C}$ is an arbitrary logarithmic function and $l:(0.1)^{2} \rightarrow \mathbb{C}$ is a bilogarithmic function. Thus, we will call it a logarithm-type functional equation

In addition, Elfen, Riedel and Sahoo [7] solved a functional equation

$$
f(p r, q s)+f(s p, r q)=2 f(p, q)+2 f(r, s)
$$

on semigroup $G$. Its solution type of $f$ on $\overline{\mathrm{G}}$ is given by

$$
f(p, q)=A(p)+A(q)+\psi\left(p q^{-1}, p q^{-1}\right)
$$

where $A: G \rightarrow \mathbb{C}$ is a homomorphism and $\psi: G \rightarrow \mathbb{C}$ is a symmetric bi-homomorphism.

Now we consider the logarithm-type functional equation given by

$$
\frac{1}{2}[f(p r, q s)+f(p s, q r)]=f(p, q)+f(r, s) .
$$

For example, if $f(x, y)=\ln x y$, then $f$ is a solution of the equation (2). In this paper, we investigate the hyperstability and stability of the functional equation (2). Namely, we prove that if $f$ satisfies a stability inequality for the equation (2), then it is also a solution of this equation and also we can find an another solution of it which has an $\varepsilon$-error bound for $f$.

\section{Hyperstability of the logarithm-type functional equation}

In this section, we investigate the hyperstability of the equation (2). Throughout this section, let $(G, \cdot)$ denote a noncommutative semigroup, $X$ a real normed space, and $\mathbb{R}$ the set of real numbers. And let $\mathbb{R}_{+}$denote the set of positive real numbers.

Theorem 1. Let $\varepsilon: G^{2} \times G^{2} \longrightarrow \mathbb{R}$ be a function such that there exists a sequence $u_{k} \in G$ that satisfies

$$
\lim _{k \rightarrow \infty} \varepsilon\left(u_{k}(p, q),(r, s)\right)=0
$$

for all $p, q, r, s \in G$. Assume that $f: G \times G \longrightarrow X$ satisfies the stability inequality

$$
\left\|\frac{1}{2}[f(p r, q s)+f(p s, q r)]-f(p, q)-f(r, s)\right\| \leq \varepsilon((p, q),(r, s))
$$

for all $p, q, r, s \in G$. Then,

$$
\frac{1}{2}[f(p r, q s)+f(p s, q r)]=f(p, q)+f(r, s) .
$$

Proof. Define a function $F: G^{2} \times G^{2} \longrightarrow X$ by

$$
F((p, q),(r, s))=f(p, q)+f(r, s)-\frac{1}{2}[f(p r, q s)+f(p s, q r)] .
$$

Then, for all $p, q, r, s, v, w \in G$, we have

$$
\begin{aligned}
F( & (p, q),(r, s))+\frac{1}{2}[F((p r, q s),(v, w))+F((p s, q r),(v, w))] \\
= & f(p, q)+f(r, s)+f(v, w) \\
& -\frac{1}{2}[f(p r v, q s w)+f(p r w, q s v)+f(p s v, q r w)+f(p s w, q r v)] .
\end{aligned}
$$


And also, for all $p, q, r, s, v, w \in G$, we have

$$
\begin{aligned}
& F((r, s),(v, w))+\frac{1}{2}[F((p, q),(r v, s w))+F((p, q),(r w, s v))] \\
& =f(p, q)+f(r, s)+f(v, w) \\
& \quad-\frac{1}{2}[f(p r v, q s w)+f(p s w, q r v)+f(p r w, q s v)+f(p s v, q r w)] .
\end{aligned}
$$

Thus, $F$ satisfies the following functional equation

$$
\begin{aligned}
& F((p, q),(r, s))+\frac{1}{2}[F((p r, q s),(v, w))+F((p s, q r),(v, w))] \\
& \quad=F((r, s),(v, w))+\frac{1}{2}[F((p, q),(r v, s w))+F((p, q),(r w, s v))] .
\end{aligned}
$$

By (3), we get

$$
\|F((p, q),(r, s))\| \leq \varepsilon((p, q),(r, s)),
$$

and with the assumed sequence $\left\{u_{k}\right\}$, we obtain

$$
\lim _{k \rightarrow \infty} F\left(u_{k}(p, q),(r, s)\right) \leq \lim _{k \rightarrow \infty} \varepsilon\left(u_{k}(p, q),(r, s)\right)
$$

for all $p, q, r, s \in G$.

The equation (4) implies

$$
\begin{aligned}
F((r, s),(v, w)) & \\
= & F((p, q),(r, s))+\frac{1}{2}[F((p r, q s),(v, w))+F((p s, q r),(v, w))] \\
& -\frac{1}{2}[F((p, q),(r v, s w))+F((p, q),(r w, s v))] .
\end{aligned}
$$

Let $r, s, v, w, p_{0}, q_{0}$ be fixed. Applying the norm and substituting $p=u_{k} p_{0}, q=u_{k} q_{0}$ in (6), and as $k \rightarrow \infty$, respectively, we obtain

$$
\begin{aligned}
& \left.\| \lim _{k \rightarrow \infty} F(r, s),(v, w)\right) \| \\
& =\| \lim _{k \rightarrow \infty}\left[F\left(u_{k}\left(p_{0}, q_{0}\right),(r, s)\right)+\frac{1}{2}\left[F\left(u_{k}\left(p_{0} r, q_{0} s\right),(v, w)\right)+F\left(u_{k}\left(p_{0} s, q_{0} r\right),(v, w)\right)\right]\right. \\
& \left.\quad-\frac{1}{2}\left[F\left(u_{k}\left(p_{0}, q_{0}\right),(r v, s w)\right)+F\left(u_{k}\left(p_{0}, q_{0}\right),(r w, s v)\right)\right]\right] \| .
\end{aligned}
$$

By applying of (5) and the triangle inequalities, we obtain

$$
\begin{aligned}
& \| F(r, s),(v, w)) \| \\
& \leq\left|\lim _{k \rightarrow \infty}\left[\varepsilon\left(u_{k}\left(p_{0}, q_{0}\right),(r, s)\right)+\frac{1}{2}\left[\varepsilon\left(u_{k}\left(p_{0} r, q_{0} s\right),(v, w)\right)+\varepsilon\left(u_{k}\left(p_{0} s, q_{0} r\right),(v, w)\right)\right]\right]\right| \\
& \quad+\left|\lim _{k \rightarrow \infty} \frac{1}{2}\left[\varepsilon\left(u_{k}\left(p_{0}, q_{0}\right),(r v, s w)\right)+\varepsilon\left(u_{k}\left(p_{0}, q_{0}\right),(r w, s v)\right)\right]\right| .
\end{aligned}
$$

Hence, we obtain from the assumed sequence $\left\{u_{k}\right\}$ the required result

$$
F((r, s),(v, w))=0
$$

for any $r, s, v, w \in G$.

Corollary 2. Assume that $f: \mathbb{R}_{+} \times \mathbb{R}_{+} \longrightarrow X$ satisfies the stability inequality

$$
\left\|\frac{1}{2}[f(p r, q s)+f(p s, q r)]-f(p, q)-f(r, s)\right\| \leq \frac{r s}{p q} \quad \text { or } \quad \text { pqrs }
$$

for all $p, q, r, s \in R_{+}$. Then,

$$
\frac{1}{2}[f(p r, q s)+f(p s, q r)]=f(p, q)+f(r, s) .
$$

Proof. Let $\varepsilon((p, q),(r, s))=\frac{r s}{p q}$ and $u_{k}=a^{k}$ for $a>1$, or $\varepsilon((p, q),(r, s))=p q r s$ and $u_{k}=a^{k}$ for $0<a<1$. Then, we obtain

so the result holds.

$$
\lim _{k \rightarrow \infty} \varepsilon\left(u_{k}(p, q),(r, s)\right)=0,
$$




\section{Stability of the logarithm-type functional equation}

In this section, we investigate the stability of the equation (2). Throughout this section, let $(G, \cdot)$ denote a commutative semigroup, $N$ the set of natural numbers, and $X$ a Banach space.

Theorem 3. Let $\varepsilon>0$. Assume that $f: G \times G \longrightarrow X$ satisfies the stability inequality

$$
\left\|\frac{1}{2}[f(p r, q s)+f(p s, q r)]-f(p, q)-f(r, s)\right\| \leq \varepsilon
$$

for all $p, q, r, s \in G$. Then there exists a function $F: G \times G \longrightarrow X$ such that

$$
\frac{1}{2}[F(p r, q s)+F(p s, q r)]=F(p, q)+F(r, s)
$$

and $\|F(p, q)-f(p, q)\| \leq \frac{39 \varepsilon}{40}$ for any $p, q \in G$, where $F$ is defined by

$$
\begin{aligned}
F(p, q):= & \lim _{n \rightarrow \infty}\left[\frac{1}{2^{2^{n}}} f\left(p^{2^{n}}, q^{2^{n}}\right)\right. \\
& \left.+\left(\sum_{i=0}^{n-2} \frac{1}{2^{i} \cdot 2^{2^{n-i}}}+\frac{1}{2^{n+1}}\right) f\left((p q)^{2^{n-1}},(p q)^{2^{n-1}}\right)\right]
\end{aligned}
$$

for any $p, q, r, s \in G$.

Proof. Letting $r=p, s=q$ in (8) and dividing it by 2, we have

$$
\left\|\frac{1}{2^{2}}\left[f\left(p^{2}, q^{2}\right)+f(p q, p q)\right]-f(p, q)\right\| \leq \frac{\varepsilon}{2} .
$$

And also, letting $p=q=r=s$ in (8) and dividing it by 2 , we have

$$
\left\|\frac{1}{2} f\left(p^{2}, p^{2}\right)-f(p, p)\right\| \leq \frac{\varepsilon}{2} .
$$

Let us show that the following inequality holds for every $n \in N$ :

$$
\left\|\frac{1}{2} f\left(p^{2^{n}}, p^{2^{n}}\right)-f\left(p^{2^{n-1}}, p^{2^{n-1}}\right)\right\| \leq \frac{\varepsilon}{2} .
$$

Replacing $p$ by $p^{2}$ and $q$ by $q^{2}$ in (9) respectively, and dividing $2^{2}$, we have

$$
\left\|\frac{1}{2^{2^{2}}}\left[f\left(p^{2^{2}}, q^{2^{2}}\right)+f\left((p q)^{2},(p q)^{2}\right)\right]-\frac{f\left(p^{2}, q^{2}\right)}{2^{2}}\right\| \leq \frac{\varepsilon}{2 \cdot 2^{2}} .
$$

Thus by (9),(10), and (12), we have

$$
\begin{aligned}
& \left\|\frac{1}{2^{2^{2}}} f\left(p^{2^{2}}, q^{2^{2}}\right)+\left(\frac{1}{2^{2^{2}}}+\frac{1}{2^{2}} \frac{1}{2}\right) f\left((p q)^{2},(p q)^{2}\right)-f(p, q)\right\| \\
& \leq\left\|\frac{1}{2^{2}}\left[f\left(p^{2^{2}}, q^{2^{2}}\right)+f\left((p q)^{2},(p q)^{2}\right)\right]-\frac{f\left(p^{2}, q^{2}\right)}{2^{2}}\right\| \\
& \quad+\left\|\frac{1}{2^{2}}\left[f\left(p^{2}, q^{2}\right)+f(p q, p q)\right]-f(p, q)\right\| \\
& \quad+\frac{1}{2^{2}}\left\|\frac{f\left((p q)^{2},(p q)^{2}\right)}{2}-f(p q, p q)\right\| \\
& \leq \frac{\varepsilon}{2 \cdot 2^{2}}+\frac{\varepsilon}{2}+\frac{\varepsilon}{2 \cdot 2^{2}}=\frac{\varepsilon}{2}+\frac{\varepsilon}{2^{2}} .
\end{aligned}
$$

In addition, by letting $p$ by $p^{2^{n-1}}$ and $q$ by $q^{2^{n-1}}$ in (9), and dividing $2^{2^{n-1}}$, the following inequality holds for every $n \in N$ :

$$
\begin{aligned}
& \left\|\frac{1}{2^{2^{n}}}\left[f\left(p^{2^{n}}, q^{2^{n}}\right)+f\left((p q)^{2^{n-1}},(p q)^{2^{n-1}}\right)\right]-\frac{f\left(p^{2^{n-1}}, q^{2^{n-1}}\right)}{2^{2^{n-1}}}\right\| \\
& \leq \frac{\varepsilon}{2 \cdot 2^{2^{n-1}}} .
\end{aligned}
$$


By (10),(12), and (14), we have

$$
\begin{aligned}
& \left\|\frac{1}{2^{2^{3}}} f\left(p^{2^{3}}, q^{2^{3}}\right)+\left(\frac{1}{2^{2^{3}}}+\left(\frac{1}{2^{2^{2}}}+\frac{1}{2^{3}}\right) \frac{1}{2}\right) f\left((p q)^{2^{2}},(p q)^{2^{2}}\right)-f(p, q)\right\| \\
& \leq\left\|\frac{1}{2^{2^{3}}}\left[f\left(p^{2^{3}}, q^{2^{3}}\right)+f\left((p q)^{2^{2}},(p q)^{2^{2}}\right)\right]-\frac{f\left(p^{2^{2}}, q^{2^{2}}\right)}{2^{2^{2}}}\right\| \\
& \quad+\left\|\frac{1}{2^{2^{2}}} f\left(p^{2^{2}}, q^{2^{2}}\right)+\left(\frac{1}{2^{2^{2}}}+\frac{1}{2^{3}}\right) f\left((p q)^{2},(p q)^{2}\right)-f(p, q)\right\| \\
& \quad+\left(\frac{1}{2^{2}}+\frac{1}{2^{3}}\right)\left\|\frac{f\left((p q)^{2^{2}},(p q)^{2^{2}}\right)}{2}-f\left((p q)^{2},(p q)^{2}\right)\right\| \\
& \leq \frac{\varepsilon}{2 \cdot 2^{2^{2}}}+\frac{\varepsilon}{2}+\frac{\varepsilon}{2^{2}}+\left(\frac{\varepsilon}{2^{2^{2}}}+\frac{\varepsilon}{2^{3}}\right) \frac{1}{2} \\
& =\frac{\varepsilon}{2}+\frac{\varepsilon}{2^{2}}+\frac{\varepsilon}{2^{2}}+\frac{\varepsilon}{2^{4}} \\
& =\frac{\varepsilon}{2}+\frac{\varepsilon}{2^{2}}+\frac{\varepsilon}{2^{3}} .
\end{aligned}
$$

Note that

$$
\begin{aligned}
& \sum_{j=4}^{n} \sum_{i=2}^{j-2} \frac{\varepsilon}{2^{i} \cdot 2^{2^{j-i}}}+\sum_{i=1}^{n} \frac{\varepsilon}{2^{2^{i-1}}}+\sum_{i=3}^{n} \frac{\varepsilon}{2^{i+1}} \\
& \leq \frac{\varepsilon}{2^{2}}\left(\frac{1}{2^{2^{2}}}+\frac{1}{2^{2^{3}}}+\frac{1}{2^{2^{4}}}+\cdots\right)+\frac{\varepsilon}{2^{3}}\left(\frac{1}{2^{2^{2}}}+\frac{1}{2^{2^{3}}}+\frac{1}{2^{2^{4}}}+\cdots\right) \\
& \quad+\cdots+\frac{\varepsilon}{2^{n-1}}\left(\frac{1}{2^{2^{2}}}+\frac{1}{2^{2^{3}}}+\frac{1}{2^{2^{4}}}+\cdots\right) \\
& \quad+\frac{\varepsilon}{2}+\frac{\varepsilon}{2^{2}}+\varepsilon\left(\frac{1}{2^{2}}+\frac{1}{2^{2^{3}}}+\frac{1}{2^{2^{4}}}+\cdots\right)+\frac{\varepsilon}{2^{4}}\left(1+\frac{1}{2}+\frac{1}{2^{2}}+\frac{1}{2^{3}}+\cdots\right) \\
& =\varepsilon\left(\frac{1}{2^{2}}+\frac{1}{2^{2^{3}}}+\frac{1}{2^{2^{4}}}+\cdots\right)\left(1+\frac{1}{2^{2}}+\frac{1}{2^{3}}+\frac{1}{2^{4}}+\cdots\right) \\
& \quad+\frac{\varepsilon}{2}+\frac{\varepsilon}{2^{2}}+\frac{\varepsilon}{2^{4}}\left(1+\frac{1}{2}+\frac{1}{2^{2}}+\frac{1}{2^{3}}+\cdots\right) \\
& =\varepsilon \cdot \frac{1}{15} \cdot \frac{3}{2}+\frac{\varepsilon}{2}+\frac{\varepsilon}{2^{2}}+\frac{\varepsilon}{2^{3}} \\
& =\frac{39 \varepsilon}{40} .
\end{aligned}
$$

Suppose that the following inequality holds for $n \geq 4$ and for any $p, q \in G$ :

$$
\begin{aligned}
& \left\|\frac{1}{2^{2^{n}}} f\left(p^{2^{n}}, q^{2^{n}}\right)+\left(\sum_{i=0}^{n-2} \frac{1}{2^{i} \cdot 2^{2^{n-i}}}+\frac{1}{2^{n+1}}\right) f\left((p q)^{2^{n-1}},(p q)^{2^{n-1}}\right)-f(p, q)\right\| \\
& \quad \leq \sum_{j=4}^{n} \sum_{i=2}^{j-2} \frac{\varepsilon}{2^{i} \cdot 2^{2^{j-i}}}+\sum_{i=1}^{n} \frac{\varepsilon}{2^{2^{i-1}}}+\sum_{i=3}^{n} \frac{\varepsilon}{2^{i+1}} .
\end{aligned}
$$

Note that

$$
\sum_{i=0}^{n-1} \frac{1}{2^{i} \cdot 2^{2^{n+1-i}}}=\frac{1}{2^{2^{n+1}}}+\frac{1}{2} \sum_{i=0}^{n-2} \frac{1}{2^{i} \cdot 2^{2^{n-i}}}
$$


for all $n \in N$. Then, for any $p, q \in G$, based on (14) and (18), we obtain

$$
\begin{aligned}
& \| \frac{1}{2^{2^{n+1}}} f\left(p^{2^{n+1}}, q^{2^{n+1}}\right) \\
& +\left(\sum_{i=0}^{n-1} \frac{1}{2^{i} \cdot 2^{2^{n+1-i}}}+\frac{1}{2^{n+2}}\right) f\left((p q)^{2^{n}},(p q)^{2^{n}}\right)-f(p, q) \| \\
& \leq\left\|\frac{1}{2^{2^{n+1}}}\left[f\left({p^{2^{n+1}}}, q^{2^{n+1}}\right)+f\left((p q)^{2^{n}},(p q)^{2^{n}}\right)\right]-\frac{f\left(p^{2^{n}}, q^{2^{n}}\right)}{2^{2^{n}}}\right\| \\
& +\| \frac{1}{2^{2^{n}}} f\left(p^{2^{n}}, q^{2^{n}}\right) \\
& +\left(\sum_{i=0}^{n-2} \frac{1}{2^{i} \cdot 2^{2^{n-i}}}+\frac{1}{2^{n+1}}\right) f\left((p q)^{2^{n-1}},(p q)^{2^{n-1}}\right)-f(p, q) \| \\
& +\left(\sum_{i=0}^{n-2} \frac{1}{2^{i} \cdot 2^{2^{n-i}}}+\frac{1}{2^{n+1}}\right)\left\|\frac{f\left((p q)^{2^{n}},(p q)^{2^{n}}\right)}{2}-f\left((p q)^{2^{n-1}},(p q)^{2^{n-1}}\right)\right\| \\
& =\frac{\varepsilon}{2 \cdot 2^{2^{n}}}+\sum_{j=4}^{n} \sum_{i=2}^{j-2} \frac{\varepsilon}{2^{i} \cdot 2^{2^{j-i}}}+\sum_{i=1}^{n} \frac{\varepsilon}{2^{2^{i-1}}} \\
& +\sum_{i=3}^{n} \frac{\varepsilon}{2^{i+1}}+\left(\sum_{i=0}^{n-2} \frac{1}{2^{i} \cdot 2^{2^{n-i}}}+\frac{1}{2^{n+1}}\right) \frac{\varepsilon}{2} \\
& =\left(\sum_{j=4}^{n} \sum_{i=2}^{j-2} \frac{\varepsilon}{2^{i} \cdot 2^{2^{j-i}}}+\sum_{i=2}^{n-1} \frac{\varepsilon}{2^{i} \cdot 2^{2^{n+1-i}}}\right) \\
& +\left(\frac{\varepsilon}{2^{2^{n}}}+\sum_{i=1}^{n} \frac{\varepsilon}{2^{2^{i-1}}}\right)+\left(\sum_{i=3}^{n} \frac{\varepsilon}{2^{i+1}}+\frac{\varepsilon}{2^{n+2}}\right) \\
& =\sum_{j=4}^{n+1} \sum_{i=2}^{j-2} \frac{\varepsilon}{2^{i} \cdot 2^{2^{j-i}}}+\sum_{i=1}^{n+1} \frac{\varepsilon}{2^{2^{i-1}}}+\sum_{i=3}^{n+1} \frac{\varepsilon}{2^{i+1}} .
\end{aligned}
$$

Thus, by induction, inequality (17) holds for all $n \geq 4$ and for any $p, q \in G$. Now for $n \geq 4$, we have

$$
\begin{aligned}
& \| \frac{1}{2^{2^{n}}} f\left(p^{2^{n}}, q^{2^{n}}\right)+\left(\sum_{i=0}^{n-2} \frac{1}{2^{i} \cdot 2^{2^{n-i}}}+\frac{1}{2^{n+1}}\right) f\left((p q)^{2^{n-1}},(p q)^{2^{n-1}}\right) \\
& -\frac{1}{2^{2^{n-1}}} f\left(p^{2^{n-1}}, q^{2^{n-1}}\right) \\
& -\left(\sum_{i=0}^{n-3} \frac{1}{2^{i} \cdot 2^{2^{n-1-i}}}+\frac{1}{2^{n}}\right) f\left((p q)^{2^{n-2}},(p q)^{2^{n-2}}\right) \|
\end{aligned}
$$

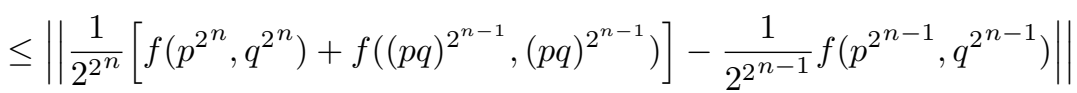

$$
\begin{aligned}
& +\sum_{i=0}^{n-3} \frac{1}{2^{i} \cdot 2^{2^{n-1-i}}}\left\|\frac{f\left((p q)^{2^{n-1}},(p q)^{2^{n-1}}\right)}{2}-f\left((p q)^{2^{n-2}},(p q)^{2^{n-2}}\right)\right\| \\
& +\left\|\frac{1}{2^{n+1}} f\left((p q)^{2^{n-1}},(p q)^{2^{n-1}}\right)-\frac{1}{2^{n}} f\left((p q)^{2^{n-2}},(p q)^{2^{n-2}}\right)\right\| \\
& \leq \frac{\varepsilon}{2 \cdot 2^{2^{n-1}}}+\sum_{i=0}^{n-3} \frac{1}{2^{i} \cdot 2^{2^{n-1-i}}} \cdot \frac{\varepsilon}{2}+\frac{\varepsilon}{2 \cdot 2^{n}} \\
& \leq \frac{\varepsilon}{2 \cdot 2^{2^{n-1}}}+\frac{1}{2^{2^{n-1}}} \sum_{i=0}^{n-3} \frac{1}{2^{i+1}} \cdot \frac{\varepsilon}{2}+\frac{\varepsilon}{2 \cdot 2^{n}} \quad \longrightarrow \quad 0
\end{aligned}
$$

as $n \rightarrow \infty$. Thus, if we let

$$
Y_{n}=\frac{1}{2^{2^{n}}} f\left(p^{2^{n}}, q^{2^{n}}\right)+\left(\sum_{i=0}^{n-2} \frac{1}{2^{i} \cdot 2^{2^{n-i}}}+\frac{1}{2^{n+1}}\right) f\left((p q)^{2^{n-1}},(p q)^{2^{n-1}}\right),
$$


then $\left\{Y_{n}\right\}$ is a Cauchy sequence due to (20), and so we can define a function $F: G \times G \longrightarrow X$ by

$$
\begin{aligned}
F(p, q):= & \lim _{n \rightarrow \infty}\left[\frac{1}{2^{2^{n}}} f\left(p^{2^{n}}, q^{2^{n}}\right)\right. \\
& \left.+\left(\sum_{i=0}^{n-2} \frac{1}{2^{i} \cdot 2^{2^{n-i}}}+\frac{1}{2^{n+1}}\right) f\left((p q)^{2^{n-1}},(p q)^{2^{n-1}}\right)\right] .
\end{aligned}
$$

Then, due to (16), (17), and (21), we have

$$
\|F(p, q)-f(p, q)\| \leq \frac{39 \varepsilon}{40} \quad \forall p, q \in G .
$$

Finally, the function $F$ defined in (21) holds the required equation (2) as follows:

$$
\begin{aligned}
& \left\|\frac{1}{2}[F(p r, q s)+F(p s, q r)]-F(p, q)-F(r, s)\right\| \\
& \leq \lim _{n \rightarrow \infty} \| \frac{1}{2} \frac{1}{2^{2^{n}}} f\left((p r)^{2^{n}},(q s)^{2^{n}}\right) \\
& +\frac{1}{2}\left(\sum_{i=0}^{n-2} \frac{1}{2^{i} \cdot 2^{2^{n-i}}}+\frac{1}{2^{n+1}}\right) f\left((p q r s)^{2^{n-1}},(p q r s)^{2^{n-1}}\right) \\
& +\frac{1}{2} \frac{1}{2^{2^{n}}} f\left((p s)^{2^{n}},(q r)^{2^{n}}\right) \\
& +\frac{1}{2}\left(\sum_{i=0}^{n-2} \frac{1}{2^{i} \cdot 2^{2^{n-i}}}+\frac{1}{2^{n+1}}\right) f\left((p q r s)^{2^{n-1}},(p q r s)^{2^{n-1}}\right) \\
& -\frac{1}{2^{2^{n}}} f\left((p)^{2^{n}},(q)^{2^{n}}\right) \\
& -\left(\sum_{i=0}^{n-2} \frac{1}{2^{i} \cdot 2^{2^{n-i}}}+\frac{1}{2^{n+1}}\right) f\left((p q)^{2^{n-1}},(p q)^{2^{n-1}}\right) \\
& -\frac{1}{2^{2^{n}}} f\left((r)^{2^{n}},(s)^{2^{n}}\right) \\
& -\left(\sum_{i=0}^{n-2} \frac{1}{2^{i} \cdot 2^{2^{n-i}}}+\frac{1}{2^{n+1}}\right) f\left((r s)^{2^{n-1}},(r s)^{2^{n-1}}\right) \| \\
& \leq \lim _{n \rightarrow \infty} \frac{1}{2^{2^{n}}} \| \frac{1}{2}\left[f\left((p r)^{2^{n}},(q s)^{2^{n}}\right)+f\left((p s)^{2^{n}},(q r)^{2^{n}}\right)\right] \\
& -f\left(p^{2^{n}}, q^{2^{n}}\right)-f\left(r^{2^{n}}, s^{2^{n}}\right) \| \\
& +\lim _{n \rightarrow \infty}\left(\sum_{i=0}^{n-2} \frac{1}{2^{i} \cdot 2^{2^{n-i}}}+\frac{1}{2^{n+1}}\right) \\
& \times \| \frac{1}{2}\left[f\left((p q r s)^{2^{n-1}},(\text { pqrs })^{2^{n-1}}\right)+f\left((p q r s)^{2^{n-1}},(\text { pqrs })^{2^{n-1}}\right)\right] \\
& -f\left((p q)^{2^{n-1}},(p q)^{2^{n-1}}\right)-f\left((r s)^{2^{n-1}},(r s)^{2^{n-1}}\right) \| \\
& \leq \lim _{n \rightarrow \infty} \frac{\varepsilon}{2^{2^{n}}}+\lim _{n \rightarrow \infty}\left(\sum_{i=0}^{n-2} \frac{1}{2^{i} \cdot 2^{2^{n-i}}}+\frac{1}{2^{n+1}}\right) \varepsilon \\
& =0 \text {. }
\end{aligned}
$$

Corollary 4. Let $\varepsilon>0$. Assume that $f: G \times G \longrightarrow X$ satisfies the stability inequality

$$
\left\|\frac{1}{2^{2}}\left[f\left(p^{2}, q^{2}\right)+f(p q, p q)\right]-f(p, q)\right\| \leq \varepsilon
$$

for all $p, q \in G$. Then there exists a function $F: G \times G \longrightarrow X$ such that

$$
\frac{1}{2^{2}}\left[F\left(p^{2}, q^{2}\right)+F(p q, p q)\right]=F(p, q)
$$

and $\|F(p, q)-f(p, q)\| \leq \frac{39 \varepsilon}{40}$ for any $p, q \in G$. 


\section{REFERENCES}

[1] T. Aoki, On the stability of the linear transformation in Banach spaces, J. Math. Soc. Japan, 2 (1950), 64-66.

[2] J. A. Baker, J. Lawrence and F. Zorzitto, The stability of the equation $f(x+y)=f(x) f(y)$, Proc. Amer. Math. Soc., 74 (1979), 242-246.

[3] D. G. Bourgin, Multiplicative transformations, Proc. Natl. Acad. Sci. USA, 36 (1950), 564-570.

[4] D. G. Bourgin, Approximately isometric and multiplicative transformations on continuous function rings, Duke Math. J., 16, (1949), 385-397.

[5] J. Brazdȩk and K. Ciepliński, Hyperstability and superstability, Abstr.Appl. Anal., Special Issue. (2013) 1-13.

[6] J. K. Chung, Pl. Kannappan, C. T. Ng and P. K. Sahoo, Measures of distance between probability distributions, J. Math. Anal. Appl., 138 (1989), 280-292.

[7] H. H. Elfen, T. Riedel and P. K. Sahoo, A variant of the quadratic functional equation on groups and an application Bull. Korean Math. Soc. 54(6), (2017), 2165-2182

[8] P. Gavruta, A Generalization of the Hyers-Ulam-Rassias Stability of Approximately Additive Mapping, J. Math. Anal. Appl., 184 (1994), 431-436.

[9] R. Ger, Superstability is not natural, Roczik Naukowo-Dydaktyczny WSP w Krakowie, Prace Mat. 159, (1994) 109-123.

[10] E. Gselmann, Hyperstability of functional equation, Acta Math. Hungar., 124(1-2), 2009, 179-188.

[11] D. H. Hyers, On the stability of the linear functional equation, Proc. Natl. Acad. Sci. 27(1941), 222-224.

[12] G. Maksa and Z. Páles, Hyperstability of a class of linear functional equations, Acta Math. Acad. Paedagog. Nyhazi. (N. S.) 17 (2001) $107-112$.

[13] Th. M. Rassias, On the stability of the linear mapping in Banach spaces, Proc. Amer. Math. Soc. 72(1978), 297-300.

[14] T. Riedel and P. K. Sahoo, On a generalization of a functional equation associated with the distance between the probability diastributions, Publ. Math. Debrecen, 46 (1995), 125-135.

[15] Sirouni and S. Kabbaj, The E-hyperstability of an Euler-Lagrange type quadratic functional equation in Banach spaces, British J. Math. Computer Science, 6(6) (2015), 481-493.

[16] S. M. Ulam, A collection of mathematical problems, 8, Interscience Publishers New York 1960. 